\title{
Ginsenoside Rh2 Mitigates Pediatric Leukemia Through Suppression of Bcl-2 in Leukemia Cells
}

\author{
Xiaoru Wang Yulin Wang \\ Department of Pediatrics, Provincial Hospital Affiliated to Shandong University, Jinan, China
}

\section{Key Words}

Pediatric leukemia • Ginsenoside Rh2 (GRh2) • Bcl-2 • MiR-21

\begin{abstract}
Background/Aims: Acute myeloid leukemia (AML) is a severe malignant cancer worldwide, in both adult and pediatric patients. Since bone marrow cell transplantation is seriously limited by the availability of the immune-paired donor sources, the therapy for pediatric leukemia remains challenging. Ginsenoside Rh2 (GRh2) is a well-characterized component in red ginseng, and has established therapeutic effects for different diseases, although whether GRh2 may have a therapeutic effect on pediatric leukemia has not been investigated. Methods: We examined the effects of GRh2 on the survival of mice in an acute leukemia model. We analyzed the effects of GRh2 on the cell viability of leukemia cell lines in vitro, using a CCK-8 assay and an MTT assay. We analyzed the effects of GRh2 on the apoptosis of leukemia cell lines in vitro, by flow cytometry. We analyzed the levels of Bcl-2 and microRNA-21 (miR-21) in GRh2treated leukemia cells. Prediction of binding between miR-21 and 3'-UTR of Bcl-2 mRNA was performed by a bioinformatics algorithm and confirmed by a dual luciferase reporter assay. Results: GRh2 significantly prolonged the survival of mice with pediatric leukemia. GRh2 significantly decreased the viability of leukemia cells in vitro, through induction of apoptosis. GRh2 significantly decreased the levels of an anti-apoptotic protein Bcl-2 in leukemia cells, possibly through induction of miR-21, which suppressed the translation of Bcl-2 mRNA via 3'-UTR binding. Conclusion: GRh2 may be an effective treatment for pediatric leukemia, and GRh2 may induce apoptosis of leukemia cells through miR-21-modulated suppression of $\mathrm{BCl}-2$.

\section{Introduction}

Acute myeloid leukemia (AML) is a severe malignant cancer worldwide [1]. Although detected mainly in teenagers and adults, pediatric cases comprise a certain percentage of newly diagnosed AML patients [1]. The etiology and pathology of AML in children are not completely determined [2-5]. However, it is known that some AML in children with Down syndrome (DS) is characterized by a founding GATA1 mutation that cooperates with trisomy

Yulin Wang

KARGER 125
Department of Pediatrics, Provincial Hospital Affiliated to Shandong University, 324 Jingwu Road, Jinan 250021, (China)

Tel. / Fax+86053181958767, E-Mail xiaoru_wang14@163.com 


\section{Cellular Physiology Cell Physiol Biochem 2015;37:641-650

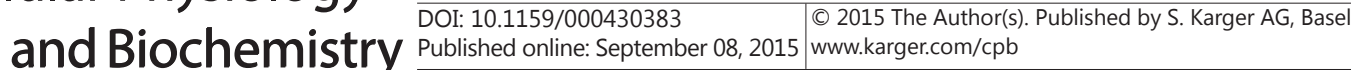 \\ Wang/Wang: GRh2 Induces Apoptosis of Pediatric Leukemia Cells}

21 , followed by the acquisition of additional somatic mutations in epigenetic and kinase signaling genes [6-8]. Clinically this manifests as a transient over-proliferation of myeloid cells at the early years of life, followed by spontaneous resolution and the subsequent development into AML in some patients with a latency of one to five years [6-8]. Likewise, there are other types of AML, the causes of which are not understood. Moreover, current therapies rely largely on bone marrow cell transplantation, which is seriously limited by the availability of immune-paired donor sources $[9,10]$. Hence, development of efficient therapy independent of bone marrow transplantation may substantially improve therapeutic outcomes.

Ginsenoside Rh2 (GRh2) is a well-characterized component in red ginseng, and has definite therapeutic effects on inflammation [11], abnormal vascularization [12], and some cancers [13-16], although the underlying mechanisms are still ill-defined. Nevertheless, whether GRh2 may be an effective treatment for pediatric leukemia has not been investigated.

MicroRNAs (miRNAs) are non-coding small RNAs of about 22 nucleotides, and have been found to regulate the expression of one third of all genes post-transcriptionally through base-pairing with the $3^{\prime}$-untranslated region (3'-UTR) of target mRNAs. Cancer initiation and progression have been associated with abnormal and aberrant expression of some miRNAs [17-22]. Specifically, miRNA-21 (miR-21) has been shown to be an aberrantly expressed miRNAs in early malignant lesions in some type of cancer [23-27]. Nevertheless, the mechanism by which miR-21 regulates the growth of PDAC is still not completely understood.

In our previous report, we showed that MMP13 is a key regulator of metastasis of pediatric multiple myeloma [28]. Here, we examined the effects of GRh2 on the survival of mice in an acute leukemia model. We analyzed the effects of GRh2 on the cell viability of leukemia cell lines in vitro, using CCK-8 assay and MTT assay. We analyzed the effects of GRh2 on the apoptosis of leukemia cell lines in vitro, by flow cytometry. We analyzed the levels of Bcl-2 and microRNA-21 (miR-21) in GRh2-treated leukemia cells. Prediction of binding between miR-21 and 3'-UTR of Bcl-2 mRNA was performed by a bioinformatics algorithm and confirmed by a dual luciferase reporter assay. We found that GRh2 significantly increased the survival time of mice with pediatric leukemia. GRh2 significantly decreased the viability of leukemia cells in vitro, through induction of apoptosis. GRh2 significantly decreased the levels of an anti-apoptotic protein Bcl-2 in leukemia cells, possibly through induction of miR-21, which suppressed the translation of Bcl-2 mRNA via 3'-UTR binding. Thus, our data suggest that GRh2 may be an effective treatment for pediatric leukemia, and GRh2 may induce apoptosis of leukemia cells through miR-21-modulated suppression of Bcl-2.

\section{Materials and Methods}

\section{Mouse leukemia and GRh2 treatment}

Four-week-old NOD.Cg-Prkdcscid Il2rgtm1Wjl/SzJ (NOD-SCID-gamma) mice were obtained from the Jackson Laboratory (Bar Harbor, ME, USA) or bred in-house and maintained under sterile conditions. Animals were irradiated with 200 cGy using a RS-2000 irradiator (Rad Source, Suwanee, GA, USA), and immediately received intraperitoneal injection of GRh2 $(1 \mathrm{mg} / \mathrm{kg}$ body weight, Weikeqi Bioscience, China) in $0.3 \mathrm{ml}$ sterile PBS. The control mice received $0.3 \mathrm{ml}$ sterile PBS. Mice were monitored daily and recorded survival twice weekly. All experiments were conducted under the supervision of the facility's Institutional Animal Care and Use Committee according to an Institutional Animal Care and Use Committee-approved protocol.

Cell line culture and transfection

Kasumi-1 and U-937 were two leukemia cell lines obtained from the American Type Culture Collection (Manassas, VA, USA), and were both maintained in RPMI 1640 media (Sigma-Aldrich, St Louis, MO, USA) supplemented with 10\% fetal bovine serum (FBS, Invitrogen, Carlsbad, CA, USA) and 1\% penicillinstreptomycin (Invitrogen) in a humidified chamber with $5 \% \mathrm{CO}_{2}$ at $37^{\circ} \mathrm{C}$. The Kasumi-1 cell line was 


\section{Cellular Physiology Cell Physiol Biochem 2015;37:641-650

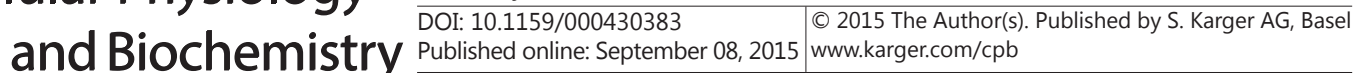 \\ Wang/Wang: GRh2 Induces Apoptosis of Pediatric Leukemia Cells}

established from the peripheral blood of an acute myeloid leukemia (AML) patient of 7 year-old male Japanese individual [29]. The U-937 cell line was derived by Sundstrom and Nilsson in 1974 from malignant cells obtained from the pleural effusion of a 37 year-old male patient with histiocytic lymphoma [30]. These two lines have been widely used for studying different subtype of leukemia.

MiRNAs mimics (miR-21) and miRNAs antisense oligonucleotides (as-miR-21) were obtained from Origene (Beijing, China). A null sequence was transfected as a control (null). The sequences were: miR-21 sequence: 5'-UAGCUUAUCAGACUGAUGUUGA-3', miR-21 antisense sequence (as-miR-21): 5'-UCAACAUCAGUCUGAUAAGCUA-3', control null sequence: 5'-UUGUACUACACAAAAGUAAUG-3'. These plasmids were transfected into cultured Kasumi- 1 and U-937 cells at a concentration of 50nmol/l using Lipofectamine 2000, according to the manufacturer's instructions (Invitrogen). The plasmids also contained a GFP reporter to allow determination of transfection efficiency, which was nearly $100 \%$ in the current study.

MiRNA target prediction and 3'-UTR luciferase-reporter assay

MiRNAs targets were predicted as has been described before, using the algorithms TargetSan (https:// www.targetscan.org) [31]. The Bcl-2 3'-UTR reporter plasmid (pRL-Bcl-2) was purchased from Creative Biogene (Shirley, NY, USA). Mir-21-modified Kasumi-1 and U-937 cells were transfected with pRL-Bcl-2 by Lipofectamine $2000\left(5 \times 10^{4}\right.$ cells per well). Cells were collected 48 hours after transfection for assay using the dual-luciferase reporter assay system gene assay kit (Promega), according to the manufacturer's instructions.

Quantitative RT-PCR (RT-qPCR)

MiRNA and total RNA were extracted from resected specimen from the patients or from cultured cells with miRNeasy mini kit or RNeasy kit (Qiagen, Hilden, Germany), respectively. Complementary DNA (cDNA) was randomly primed from $2 \mu \mathrm{g}$ of total RNA using the Omniscript reverse transcription kit (Qiagen). RTqPCR was subsequently performed in triplicate with a 1:4 dilution of cDNA using the Quantitect $\mathrm{SyBr}$ green PCR system (Qiagen) on a Rotorgene 6000 series PCR machine. Quantitative PCR (RT-qPCR) were performed in duplicates with QuantiTect SYBR Green PCR Kit (Qiagen). All primers were purchased from Qiagen. Data were collected and analyzed with the Rotorgene software accompanying the PCR machine, using $2^{-\Delta \Delta C t}$ method for quantification of the relative mRNA expression levels. Values of genes were first normalized against $\alpha$-tubulin, and then compared to controls.

Cell counting kit-8 (CCK-8) assay

The CCK-8 detection kit (Sigma-Aldrich) was used to measure cell viability according to the manufacturer's instructions. Briefly, cells were seeded in a 96-well microplate at a density of $5 \times 10^{4} / \mathrm{ml}$. After $24 \mathrm{~h}$, cells were treated with resveratrol. Subsequently, CCK-8 solution $(20 \mathrm{ml} /$ well $)$ was added and the plate was incubated at $37^{\circ} \mathrm{C}$ for $2 \mathrm{~h}$. The viable cells were counted by absorbance measurements with a monochromator microplate reader at a wavelength of $450 \mathrm{~nm}$. The optical density value was reported as the percentage of cell viability in relation to the control group (set as 100\%).

\section{MTT assay}

For assay of viable cell number, $5 \times 10^{3}$ cell per well were seeded into 96 well-plate and subjected to a Cell Proliferation Kit (MTT, Roche, Indianapolis, IN, USA), according to the instruction of the manufacturer. The MTT assay is a colorimetric assay for assessing viable cell number, taking advantage that NADPH-dependent cellular oxidoreductase enzymes in viable cells reduce the tetrazolium dye 3-(4,5-dimethylthiazol-2-yl)2,5-diphenyltetrazolium bromide (MTT) to its insoluble formazan in purple readily being quantified by absorbance value (OD) at $570 \mathrm{~nm}$ in a microtiter plate reader (Promega, Fitchburg, WI, USA). Experiments were performed 5 times.

\section{Apoptosis assay}

For analysis of cell proliferation, the cultured cells were dissociated and re-suspended at a density of $10^{6}$ cells $/ \mathrm{ml}$ in PBS. After double staining with FITC-Annexin V and propidium iodide (PI) from a FITC Annexin V Apoptosis Detection Kit I (Becton-Dickinson Biosciences, San Jose, CA, USA), cells were analyzed using FACScan flow cytometer (Becton-Dickinson Biosciences) equipped with Cell Quest software (BectonDickinson Biosciences) for determination of Annexin V+ PI- apoptotic cells. 


\section{Cellular Physiology Cell Physiol Biochem 2015;37:641-650

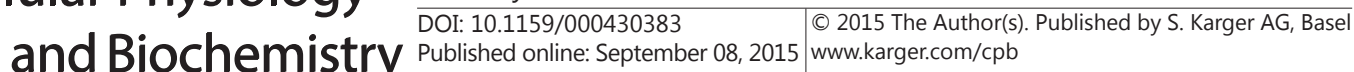 \\ Wang/Wang: GRh2 Induces Apoptosis of Pediatric Leukemia Cells}

Western blot

Total Protein was extracted from the cultured cells by RIPA buffer (Sigma-Aldrich). Equal amount of proteins was loaded in the gel. Primary antibodies for Western Blot are rabbit anti-Bcl- 2 and anti- $\alpha$ tubulin (all purchased from Cell Signaling, St Jose, LA, USA). Secondary antibody is HRP-conjugated antirabbit (Jackson ImmunoResearch Labs, West Grove, PA, USA). The protein levels were first normalized to $\alpha$-tubulin, and then normalized to control. Densitometry of Western blots was quantified with NIH ImageJ software (Bethesda, MA, USA).

\section{Statistical analysis}

All statistical analyses were carried out using the SPSS 18.0 statistical software package. All values in cell and animal studies are depicted as mean \pm standard deviation and are considered significant if $\mathrm{p}<0.05$. All data were statistically analyzed using one-way ANOVA with a Bonferoni correction, followed by Fisher' Exact Test for comparison of two groups. The survival of the mice was determined by Kaplan-Meier analysis.

\section{Result}

\section{GRh2 significantly prolongs the survival of mice with pediatric leukemia}

Four week-old male NOD/SCID mice ( $=20$ ) were irradiated with 200 cGy, and received an intraperitoneal injection of GRh2 (1mg/kg body weight) in $0.3 \mathrm{ml}$ sterile PBS $(\mathrm{N}=10)$. The control mice received $0.3 \mathrm{ml}$ sterile PBS $(\mathrm{N}=10)$. Mice were monitored daily and recorded survival twice weekly. We found that GRh2 treatment significantly prolonged the survival of mice with pediatric leukemia, analyzed by Kaplan-Meier curves (Fig. 1).

GRh2 significantly decreases the viability of leukemia cells in vitro

Then, we used two leukemia cell lines Kasumi-1 and U-937, to analyze the effects of GRh2 on cell growth. We found that GRh2 dose-dependently decreased the viable Kasumi-1 cells in either CCK-8 assay (Fig. 2A), or MTT assay (Fig. 2B). Similarly, we found that GRh2 dose-dependently decreased the viable U-937 cells in either a CCK-8 assay (Fig. 2C), or an MTT assay (Fig. 2D). Thus, these data suggest that GRh2 significantly decreases the viability of leukemia cells in vitro.

GRh2 significantly increases the apoptosis of leukemia cells in vitro

To determine whether the decreases in leukemia cells by GRh2 were mediated through modulation of cell apoptosis, we used a flow cytometry-based apoptosis assay. We found that GRh2 dose-dependently increased the apoptosis of Kasumi-1 cells, shown by quantification

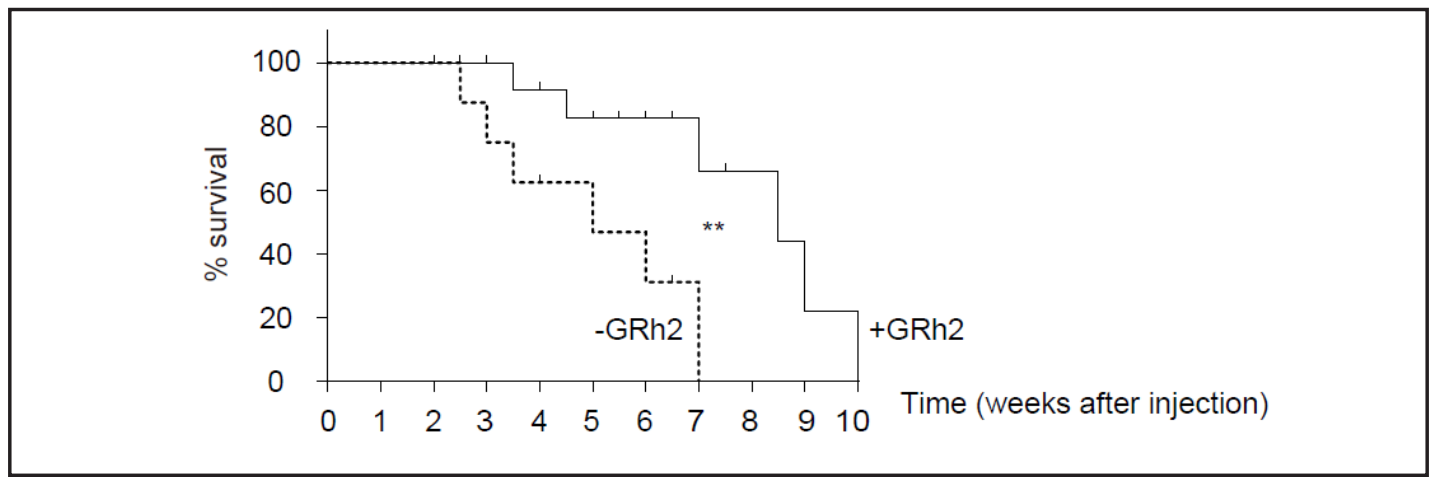

Fig. 1. GRh2 significantly prolongs the survival of mice with pediatric leukemia. Four week-old male NOD/ SCID mice ( $\mathrm{N}=20)$ were irradiated with 200 cGy, and received an intraperitoneal injection of GRh2 (1mg/ $\mathrm{kg}$ body weight) in $0.3 \mathrm{ml}$ sterile PBS $(\mathrm{N}=10 ;+\mathrm{GRh} 2)$. The control mice received $0.3 \mathrm{ml}$ sterile $\mathrm{PBS}(\mathrm{N}=10$; -GRh2). Mice were monitored daily and recorded survival twice weekly. We found that GRh2 treatment significantly prolonged the survival of mice with pediatric leukemia, analyzed by Kaplan-Meier curves. $* * \mathrm{p}<0.01 . \mathrm{N}=10$. 

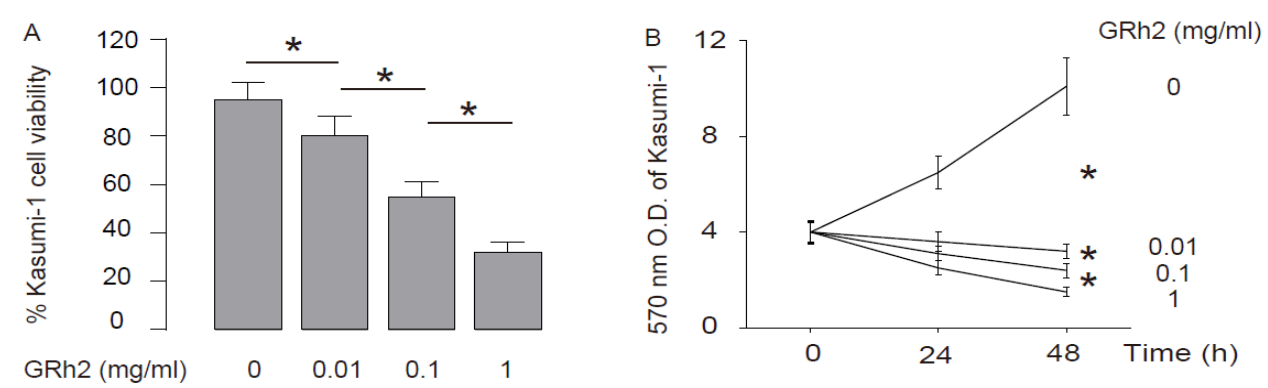

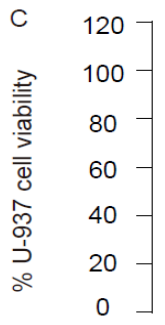
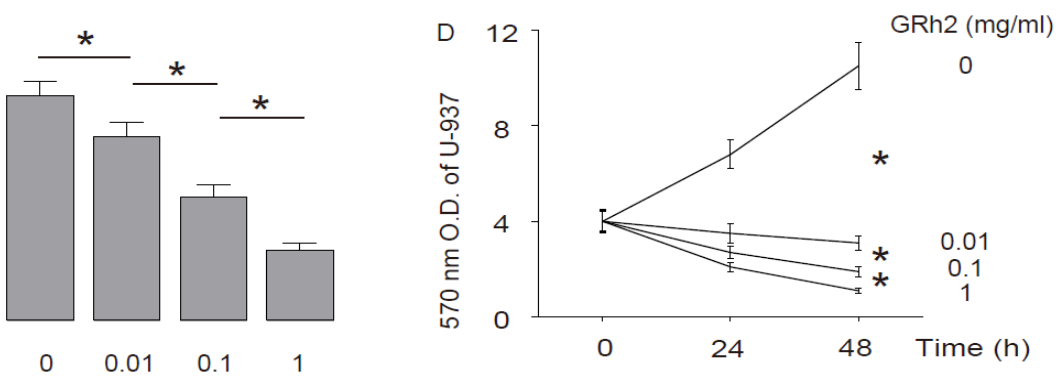

Fig. 2. GRh2 significantly decreases the viability of leukemia cells in vitro. We used two leukemia cell lines Kasumi-1 and U-937, to analyze the effects of GRh2 on cell growth. (A-B) GRh2 dose-dependently decreased the viable Kasumi-1 cells in either a CCK-8 assay (A), or an MTT assay (B). (C-D) GRh2 dose-dependently decreased the viable U-937 cells in either a CCK-8 assay (C), or an MTT assay (D). ${ }^{*} p<0.05$. N=5.

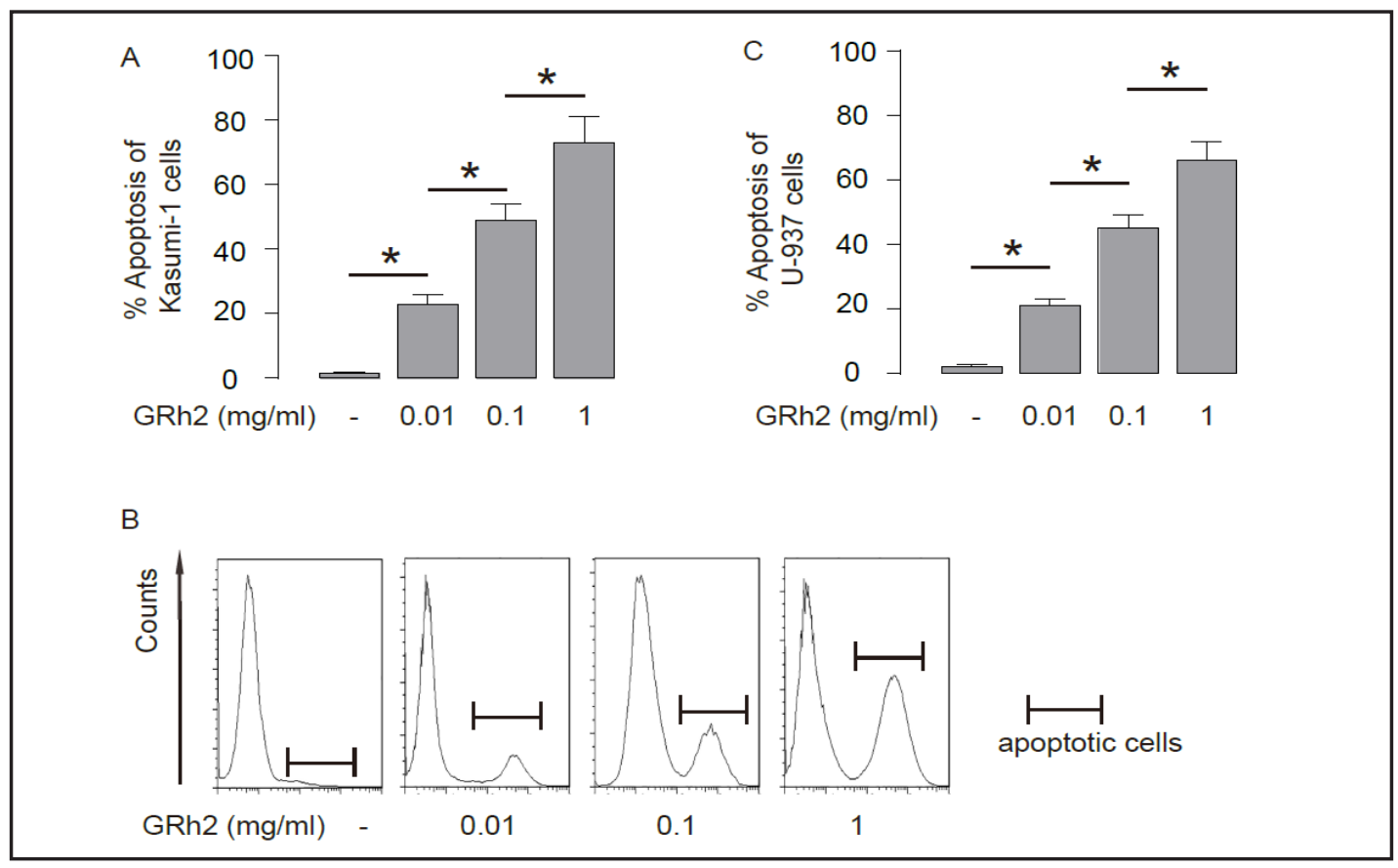

Fig. 3. GRh2 significantly increases the apoptosis of leukemia cells in vitro. (A-B) GRh2 dose-dependently increased the apoptosis of Kasumi-1 cells, shown by quantification (A), and by representative flow charts (B). (C) GRh2 dose-dependently increased the apoptosis of U-937 cells. *p $<0.05$. N=5.

(Fig. 3A), and by representative flow charts (Fig. 3B). Similarly, GRh2 dose-dependently increased the apoptosis of U-937 cells (Fig. 3C)). Hence, GRh2 significantly decreases the viability of leukemia cells in vitro, through induction of apoptosis. 


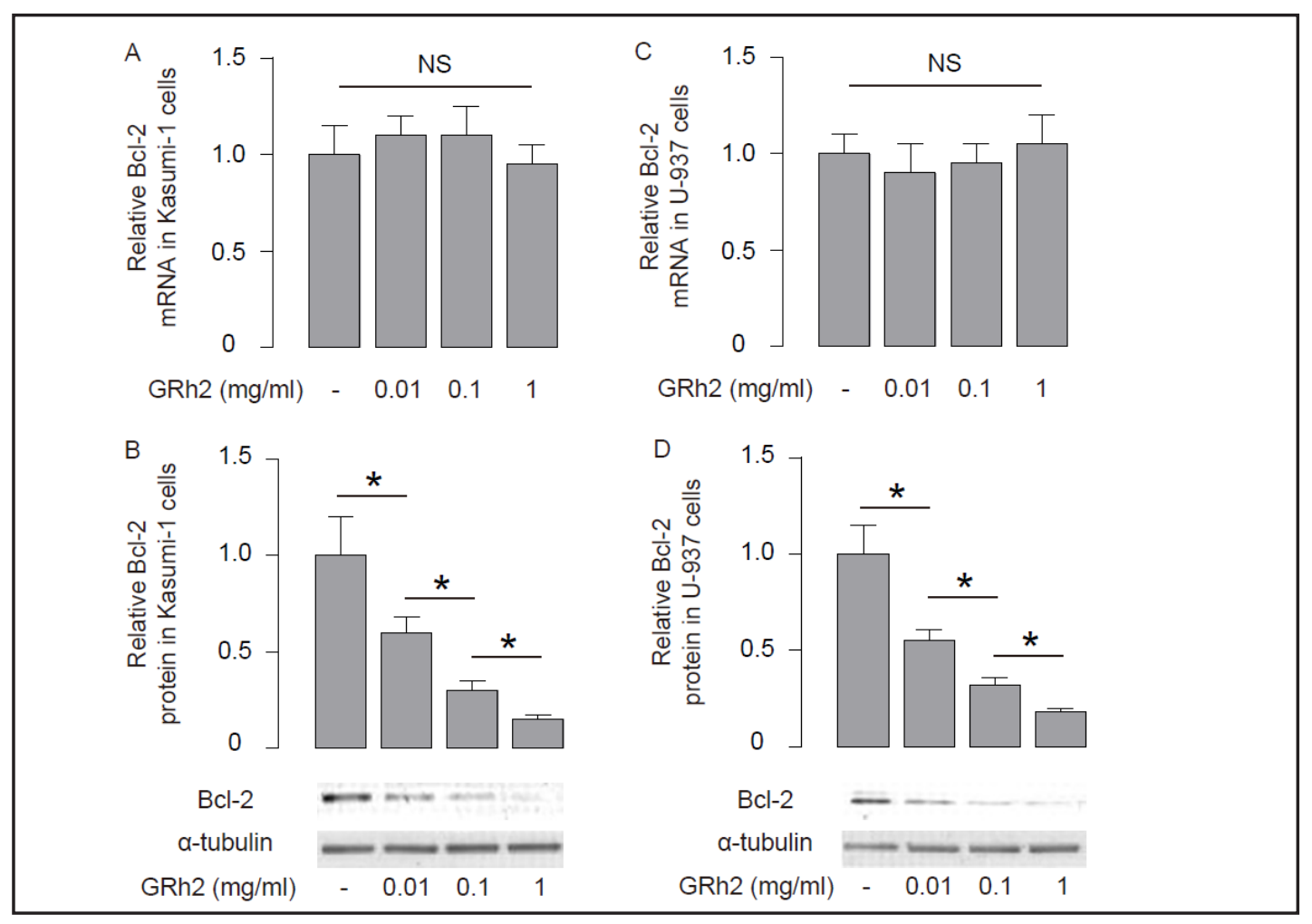

Fig. 4. GRh2 significantly decreases the levels of Bcl-2 in leukemia cells. (A-B) Bcl-2 levels in Kasumi-1 cells treated with GRh2 by mRNA (A), and by protein (B). (C-D) Bcl-2 levels in U-937 cells treated with GRh2 by mRNA (A), and by protein (B). ${ }^{*} \mathrm{p}<0.05$. NS: non-significant. $\mathrm{N}=5$.

GRh2 significantly decreases the levels of Bcl-2 in leukemia cells

We then evaluated the levels of apoptosis-associated proteins in GRh2-treated leukemia cells. Specifically, we found that although the Bcl-2 transcripts in Kasumi-1 cells did not change by GRh2 (Fig. 4A), the protein level of Bcl-2 in Kasumi-1 cells was significantly decreased by GRh2 treatment (Fig. 4B). Similarly, although the Bcl-2 transcripts in U-937 cells did not change by GRh2 (Fig. 4C), the protein levels of Bcl-2 in U-937 cells were significantly decreased by GRh2 treatment (Fig. 4D). These data suggest that the translation of Bcl-2 in leukemia cells may be regulated at the post-transcriptional level by GRh2.

GRh2 significantly decreases the levels of Bcl-2 in leukemia cells, possibly through induction of miR-21, which suppresses the translation of Bcl-2 mRNA via 3'-UTR binding

Since miRNAs are well-known to be major post-transcriptional regulators, we hypothesized that GRh2 may alter specific miRNAs that target Bcl-2 mRNA. We thus screened all Bcl-2 mRNA-targeting miRNAs, and found that miR-21 targeted 3'-UTR of Bcl-2 mRNA at one binding site of base pair $720^{\text {th }}$ to $726^{\text {th }}$ (Fig. 5A). Moreover, GRh2 dose-dependently increased the levels of miR-21 in Kasumi- 1 cells (Fig. 5B), and in U-937 cells (Fig. 5C). Thus, the intact 3'-UTR of Bcl-2 mRNA was cloned into luciferase reporter plasmids, and used for transfection of miR-21-modified Kasumi-1 and U-937 cells (Fig. 5D). We found that miR-21 significantly reduced the luciferase activity of 3'-UTR reporter for Bcl-2, while as-miR-21 significantly increased the luciferase activity of 3'-UTR reporter for Bcl-2, in both Kasumi-1 and U-937 cells (Fig. 5E). These data suggest that miR-21 targets Bcl-2 mRNA to suppress its translation. Together, we showed that GRh2 may induce apoptosis of leukemia cells through miR-21-modulated suppression of Bcl-2 (Fig. 6).

\section{KARGER}



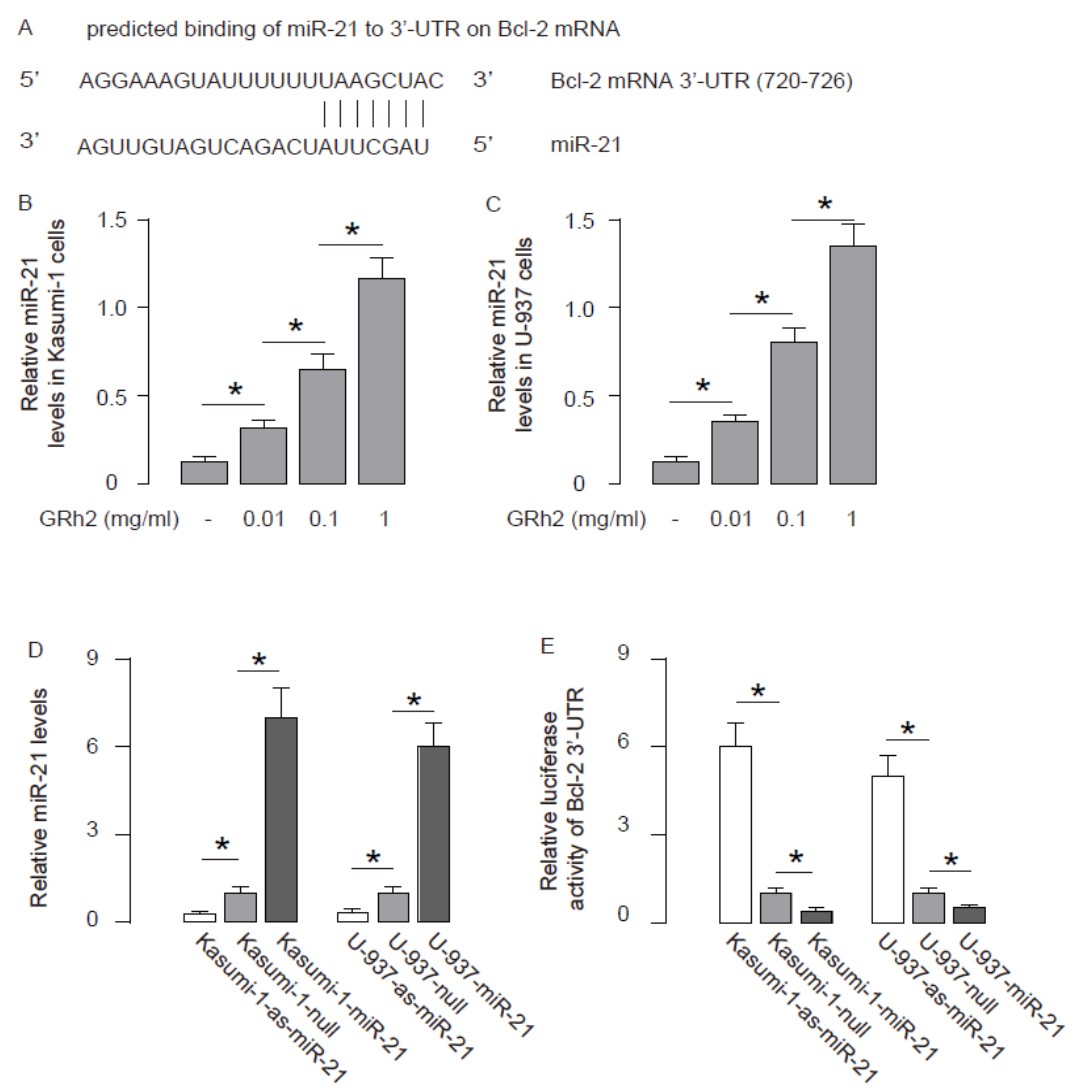

Fig. 5. GRh2 significantly decreases the levels of Bcl-2 in leukemia cells, possibly through induction of miR21, which suppresses the translation of Bcl-2 mRNA via 3'-UTR binding. (A) Bioinformatics analyses showing that miR-21 targets 3'-UTR of Bcl-2 mRNA at one binding site of base pair $720^{\text {th }}$ to $726^{\text {th }}$. (B-C) GRh2 dose-dependently increased the levels of miR-21 in Kasumi-1 cells (B), and in U-937 cells (C). (D) The levels of miR-21 in miR-21-modified Kasumi-1 and U-937 cells. (E) The intact 3'-UTR of Bcl-2 mRNA was cloned into luciferase reporter plasmids, and used for transfection of miR-21-modified Kasumi-1 and U-937 cells. The luciferase activities were quantified. ${ }^{*} \mathrm{p}<0.05$. $\mathrm{N}=5$.

Fig. 6. The schematic model. GRh2 may induce apoptosis of leukemia cells through miR-21-modulated suppression of Bcl-2.

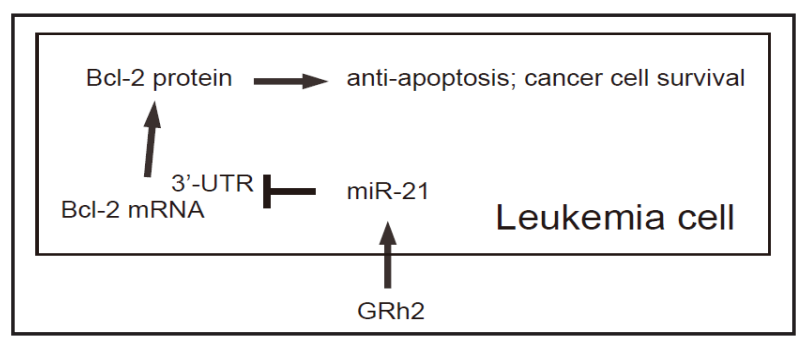

\section{Discussion}

Despite significant improvements over the past decades, 5-year survival rates for children with acute myeloid leukemia (AML) are only about 50\%, and patients with highrisk factors have an even lower predicted survival. Using current therapy, patients with relapsed AML have an extremely poor survival rate and frequently survive as little as 1 year after relapse diagnosis. Unfortunately, other than the use of all-trans retinoic acid in acute promyelocytic leukemia and the option of bone marrow cell transplantation, there have not been any new drug approvals or significant changes in AML therapy in recent years. 


\section{Cellular Physiology Cell Physiol Biochem 2015;37:641-650 \begin{tabular}{l|l|l|l|l}
\hline DOI: 10.1159/000430383 2015 The Author(s). Published by S. Karger AG, Basel & $\begin{array}{l}\text { C) } \\
\text { www.karger.com/cpb }\end{array}$
\end{tabular} \\ Wang/Wang: GRh2 Induces Apoptosis of Pediatric Leukemia Cells}

GRh2 has been shown to have therapeutic effects on various diseases. For example, in a pioneering study, Ye et al. showed that GRh2 treatment significantly alleviated dextran sulfate sodium (DSS)-induced colitis, through augmenting transforming growth factor beta (TGF-beta) signaling in colon epithelial cells [11]. In another recent report, psoriasis has been shown to be alleviated by GRh2, seemingly through suppression of VEGF-A-signalingmediated endothelial cell damages [12]. Moreover, in different cancers, GRh2 has been shown to exert anti-cancer effects through different signaling pathways, e.g. PI3k/Akt/ Fox01 or mTor [14], WNT/beta-catenin signaling [16], epidermal growth factor receptor (EGFR) signaling [13, 14], or through regulation of matrix metallopeptidases (MMPs) [15]. These previous studies prompted us to examine the effects of GRh2 on pediatric leukemia.

Here, we examined the effects of GRh2 on the survival of the mice in an acute leukemia model induced by radiation. We found that GRh2 significantly increased the survival time of mice that had developed leukemia, suggesting a therapeutic effect of GRh2. Moreover, GRh2 significantly decreased the viability of leukemia cells in vitro, through induction of apoptosis. Since previous studies have demonstrated little adverse effects of GRh2 on normal cells [1316], these data suggest that GRh2 may specifically and selectively kill leukemia cells in vivo, which substantially delays disease progression and protects the body.

Since we hypothesized that GRh2 may regulate leukemia survival through miRNAs, we performed a screening strategy in that the levels of all Bcl-2-targeting miRNAs were examined in patients' specimens and those that significantly altered between control and leukemia were selected. With this strategy, we specifically found that miR-21 was the key player in this model. Further, we found that GRh2 significantly decreased the levels of an anti-apoptotic protein Bcl-2 in leukemia cells, possibly through induction of miR-21, which suppressed the translation of Bcl-2 mRNA via 3'-UTR binding.

Interestingly, miR-21 has been recently reported to be a regulator of PI3k/Akt/FoxO1 in pancreatic cancer $[26,27]$. Based on this evidence, it may be possible that GRh2 could induce miR-21 to regulate PI3k/Akt/FoxO1 in leukemia cells, which warrants testing in future experiments. We have checked the levels of other apoptosis-associated proteins, e.g. Bid and Bak. However, it appeared that only Bcl-2 levels were predominantly affected after GRh2 treatment.

Together, our data suggest that GRh2 may be an effective treatment for pediatric leukemia, and GRh2 may induce apoptosis of leukemia cells through miR-21-modulated suppression of $\mathrm{Bcl}-2$.

\section{Disclosure Statement}

The authors have declared that no competing interests exist.

\section{References}

1 Ma J, Hua J, Sha Y, Xie Y: The effect of tlx3 expression on the prognosis of pediatric t cell acute lymphocytic leukemia--a systematic review. Tumour Biol 2014;35:8439-8443.

2 Wu X, Yang N, Zhou WH, Xu J, Chen JJ, Zheng FM, Long ZJ, Yue CF, Ai KX, Liu LL, Wan XY, Liu Q: Up-regulation of p21 inhibits trail-mediated extrinsic apoptosis, contributing resistance to saha in acute myeloid leukemia cells. Cell Physiol Biochem 2014;34:506-518.

3 Zhao S, Zhang Y, Sha K, Tang Q, Yang X, Yu C, Liu Z, Sun W, Cai L, Xu C, Cui S: Kras (g12d) cooperates with aml1/eto to initiate a mouse model mimicking human acute myeloid leukemia. Cell Physiol Biochem 2014;33:78-87.

4 Wang Y, Zhou L, Chen J, Li J, He L, Wu P, Wang M, Tong N, Zhang Z, Fang Y: Association of the 3'utr foxo3a polymorphism rs4946936 with an increased risk of childhood acute lymphoblastic leukemia in a chinese population. Cell Physiol Biochem 2014;34:325-332. 


\section{Cellular Physiology Cell Physiol Biochem 2015;37:641-650 and Biochemistry Published online: September 08, 2015 \begin{tabular}{l|l} 
(c) 2015 The Author(s). Published by S. Karger AG, Basel \\
www.karger.com/cpb
\end{tabular} \\ Wang/Wang: GRh2 Induces Apoptosis of Pediatric Leukemia Cells}

5 Liang H, Li X, Wang L, Yu S, Xu Z, Gu Y, Pan Z, Li T, Hu M, Cui H, Liu X, Zhang Y, Xu C, Guo R, Lu Y, Yang B, Shan H: Micrornas contribute to promyelocyte apoptosis in as2o3-treated apl cells. Cell Physiol Biochem 2013;32:1818-1829.

6 Arico M, Ziino O, Valsecchi MG, Cazzaniga G, Baronci C, Messina C, Pession A, Santoro N, Basso G, Conter V, Italian Association of Pediatric H, Oncology: Acute lymphoblastic leukemia and down syndrome: Presenting features and treatment outcome in the experience of the italian association of pediatric hematology and oncology (aieop). Cancer 2008;113:515-521.

7 Chang M, Raimondi SC, Ravindranath Y, Carroll AJ, Camitta B, Gresik MV, Steuber CP, Weinstein H: Prognostic factors in children and adolescents with acute myeloid leukemia (excluding children with down syndrome and acute promyelocytic leukemia): Univariate and recursive partitioning analysis of patients treated on pediatric oncology group (pog) study 8821. Leukemia 2000;14:1201-1207.

8 Liu J, Zhang X, Liu A, Liu S, Zhang L, Wu B, Hu Q: Berberine induces apoptosis in p53-null leukemia cells by down-regulating xiap at the post-transcriptional level. Cell Physiol Biochem 2013;32:1213-1224.

9 Arakawa Y, Kato M, Koh K, Hanada R: Unrelated cord blood and bone marrow transplantation in pediatric leukemia. Pediatr Int 2014;56:647-650.

10 Shaw PJ, Kan F, Woo Ahn K, Spellman SR, Aljurf M, Ayas M, Burke M, Cairo MS, Chen AR, Davies SM, Frangoul H, Gajewski J, Gale RP, Godder K, Hale GA, Heemskerk MB, Horan J, Kamani N, Kasow KA, Chan KW, Lee SJ, Leung WH, Lewis VA, Miklos D, Oudshoorn M, Petersdorf EW, Ringden O, Sanders J, Schultz KR, Seber A, Setterholm M, Wall DA, Yu L, Pulsipher MA: Outcomes of pediatric bone marrow transplantation for leukemia and myelodysplasia using matched sibling, mismatched related, or matched unrelated donors. Blood 2010;116:4007-4015.

11 Ye H, Wu Q, Zhu Y, Guo C, Zheng X: Ginsenoside rh2 alleviates dextran sulfate sodium-induced colitis via augmenting tgfbeta signaling. Mol Biol Rep 2014;41:5485-5490.

12 Zhou J, Gao Y, Yi X, Ding Y: Ginsenoside rh2 suppresses neovascularization in xenograft psoriasis model. Cell Physiol Biochem 2015;36:980-987.

13 Li S, Gao Y, Ma W, Guo W, Zhou G, Cheng T, Liu Y: Egfr signaling-dependent inhibition of glioblastoma growth by ginsenoside rh2. Tumour Biol 2014;35:5593-5598.

14 Li S, Guo W, Gao Y, Liu Y: Ginsenoside rh2 inhibits growth of glioblastoma multiforme through mtor. Tumour Biol 2015;36:2607-2612.

15 Zhang Q, Hong B, Wu S, Niu T: Inhibition of prostatic cancer growth by ginsenoside rh2. Tumour Biol 2015;36:2377-2381.

16 Liu S, Chen M, Li P, Wu Y, Chang C, Qiu Y, Cao L, Liu Z, Jia C: Ginsenoside rh2 inhibits cancer stem-like cells in skin squamous cell carcinoma. Cell Physiol Biochem 2015;36:499-508.

17 Mei Q, Li F, Quan H, Liu Y, Xu H: Busulfan inhibits growth of human osteosarcoma through mir-200 family micrornas in vitro and in vivo. Cancer Sci 2014;105:755-762.

18 Wang F, Xiao W, Sun J, Han D, Zhu Y: Mirna-181c inhibits egfr-signaling-dependent mmp9 activation via suppressing akt phosphorylation in glioblastoma. Tumour Biol 2014;35:8653-8658.

19 Liu G, Jiang C, Li D, Wang R, Wang W: Mirna-34a inhibits egfr-signaling-dependent mmp7 activation in gastric cancer. Tumour Biol 2014;35:9801-9806.

20 Ji D, Li B, Shao Q Li F, Li Z, Chen G: Mir-22 suppresses bmp7 in the development of cirrhosis. Cell Physiol Biochem 2015;36:1026-1036.

21 Wang Q, Cai J, Wang J, Xiong C, Zhao J: Mir-143 inhibits egfr-signaling-dependent osteosarcoma invasion. Tumour Biol 2014;35:12743-12748.

22 Zhang J, Wang S, Lu L, Wei G: Mir99a modulates mmp7 and mmp13 to regulate invasiveness of kaposi's sarcoma. Tumour Biol 2014;35:12567-12573.

23 Yang JS, Li BJ, Lu HW, Chen Y, Lu C, Zhu RX, Liu SH, Yi QT, Li J, Song CH: Serum mir-152, mir-148a, mir-148b, and mir-21 as novel biomarkers in non-small cell lung cancer screening. Tumour Biol 2015;36:3035-3042.

24 Yaman Agaoglu F, Kovancilar M, Dizdar Y, Darendeliler E, Holdenrieder S, Dalay N, Gezer U: Investigation of mir-21, mir-141, and mir-221 in blood circulation of patients with prostate cancer. Tumour Biol 2011;32:583-588.

25 Liu GH, Zhou ZG, Chen R, Wang MJ, Zhou B, Li Y, Sun XF: Serum mir-21 and mir-92a as biomarkers in the diagnosis and prognosis of colorectal cancer. Tumour Biol 2013;34:2175-2181.

26 Song W, Li Q, Wang L, Huang W, Wang L: Foxo1-negative cells are cancer stem-like cells in pancreatic ductal adenocarcinoma. Sci Rep 2015;5:10081. 
27 Song W, Li Q, Wang L, Wang L: Modulation of foxo1 expression by mir-21 to promote growth of pancreatic ductal adenocarcinoma. Cell Physiol Biochem 2015;35:184-190.

28 Wang X, Cao X: Regulation of metastasis of pediatric multiple myeloma by mmp13. Tumour Biol 2014;35:8715-8720.

29 Asou H, Tashiro S, Hamamoto K, Otsuji A, Kita K, Kamada N: Establishment of a human acute myeloid leukemia cell line (kasumi-1) with 8;21 chromosome translocation. Blood 1991;77:2031-2036.

30 Sundstrom C, Nilsson K: Establishment and characterization of a human histiocytic lymphoma cell line (u937). Int J Cancer 1976;17:565-577.

31 Coronnello C, Benos PV: Comir: Combinatorial microrna target prediction tool. Nucleic Acids Res 2013;41:W159-164. 